\title{
Nonlinear Analysis for Wing Rock System with Adaptive Control
}

\author{
Dongyang $\mathrm{Li}^{1}$ \\ Cranfield University, Cranfield MK43 OAL, UK \\ Nanjing University of Science and Technology, Nanjing 210094, China \\ Dmitry Ignatyev ${ }^{2}$ and Antonios Tsourdos ${ }^{3}$ \\ Cranfield University, Cranfield MK43 OAL, UK \\ Zhongyuan Wang ${ }^{4}$ \\ Nanjing University of Science and Technology, Nanjing 210094, China
}

\begin{abstract}
Adaptive control has the potential to improve the performance and reliability of aircraft. However, the inherent nonlinearity of adaptive control causes difficulty in stability and robustness verification which is critical before entry into service. In this paper, the region of attraction (ROA) estimation using sum of squares (SOS) technique is explored for adaptive control systems. The problem of wing rock suppression by a model reference adaptive control (MRAC) serves as a benchmark example to demonstrate the effectiveness of the method. Considering the potential disturbance presented in measurement signals, the operative range of aircraft must be included in the ROA of the control system. Therefore, the ROA is evaluated using SOS polynomial optimization under various combinations of design parameters in the adaptive law, including adaptation rate and sigma modification values. This gives insight into the interactions of design parameters on the adaptive control performance.
\end{abstract}

\section{Introduction}

The potential of adaptive control in improving the performance and reliability of control systems attracts researchers and engineers from different disciplines. However, the verification and validation of the flight control system regarding modeling error, uncertainty, disturbance, and time delay is not an easy task due to the inherent nonlinearity of the problem as well as the lack of rigorous analysis tools for nonlinear systems[1-6]. The fact is that safety-critical flight systems require extensive validation before entry into service. Thus, the flight control community will benefit a lot from the development of the nonlinear analysis[6].

A significant metric for a flight control system is stability. Unlike the global stability of a linear system, it is usually high-demanding or, most of the time, impossible, to guarantee stability across the whole state space for a nonlinear system. In many practical applications, global stability is also unnecessary. The actual dynamical system usually operates in a specific range of the state space due to the restrictions from the physical properties of the system. Therefore, for the engineers, it will be enough to ensure that the system works normally inside the operative range. Then, system states will not escape from the allowable range and always go back to the desired steady-state that is designed as the equilibrium of the dynamical system. The region of attraction (ROA) for the specific equilibrium happens to mark the set of initial conditions from which a trajectory will always travel back to the corresponding

${ }^{1}$ PhD student, School of Energy and Power Engineering, Nanjing University of Science and Technology.

${ }^{2}$ Senior Research Fellow, School of Aerospace, Transport and Manufacturing, Cranfield University.

${ }^{3}$ Professor, School of Aerospace, Transport and Manufacturing, Cranfield University. AIAA Senior Member.

${ }^{4}$ Professor, School of Energy and Power Engineering, Nanjing University of Science and Technology. 
equilibrium. Thus, the designer will have to work out how to ensure the operative range is included in the ROA[7,8]. This then gives rise to the problem of determining the ROA of a nonlinear system.

However, it is hard to determine the exact ROA if not impossible[7-10]. Various kinds of methods dedicated to estimating ROA, which are roughly divided into analytical and computational methods[11,12]; or into Lyapunovbased methods and non-Lyapunov-based methods[13] regarding the theory behind the methods. The analytical methods can only apply to a limited kind of relatively simple nonlinear systems. As regards the computational ones, the Monte Carlo simulation is usually used as a supplement to the analysis. The numerical simulation takes plenty of time especially for a complex and high-dimensional system, but still the results cannot be taken deterministic. The indeterminism is rooted in the sampling initial conditions. It cannot be guaranteed that the simulation distinguishes all the divergent trajectories in the final set. Another kind of computational method, instead of doing exhausted numerical integrations, solves the problem formulated by matrix inequalities, such as the Lyapunov-based estimation $[8,14,15]$. The obtained results can be deterministic since it is derived analytically but solved computationally.

Many nonlinear systems, including the adaptive system considered in this paper, are governed by polynomial dynamics. The Sum of squares (SOS) technique has the ability to solve polynomial inequalities. SOS polynomial optimization has been used previously to analyze the performance and robustness of systems described by polynomial dynamics. Computational algorithms have been developed not only for estimating ROA but also reachability sets, input-output gains, robustness with respect to uncertainty, and time-delay margin[10,16]. Applying the SOS technique for ROA estimation, this paper aims to show the ability of the techniques to analyze the stability and robustness verification for a nonlinear adaptive control system.

Wing rock is a well-known lateral-directional instability that occurs in aircraft of varying configurations and aspect ratios at a moderate to high angle of attack. The accurate modeling and control have been explored by many authors[17,18]. Therefore it serves as a classical benchmark example for the demonstration of various control methodologies[19]. In this paper, wing rock augmented with a classical model reference adaptive control (MRAC) is taken as a demonstration of the ability of the SOS technique in the nonlinear analysis of adaptive control.

The SOS techniques show great promise for ROA estimation[20] and are then modified and improved in order to gain a better approximation[12,15,21-23]. It has been applied successfully to a four-state aircraft control system for the investigation of the falling-leaf mode[24], and an eight-state aircraft model for the investigation of the spin mode[25]. A simple procedure is used in [26] for estimating the ROA of an adaptive control system. Although the SOS results are conservative, they provide rigorous proof of the stability characteristics of nonlinear systems. In its current form, the solver for the SOS optimization problem is sensitive to the dynamics of the system, such as the dimension of the system and the highest order of the polynomials. But it stands great promising in the nonlinear analysis if pursued further[6].

The paper is organized as follows. Firstly, the wing rock dynamics and the classical adaptive controller is described in Sec. III. In Sec. IV, the algorithms for ROA estimation using SOS polynomial optimization are presented. Sec. V summarizes the results of ROA analysis for an adaptive control system with respect to adaptation gain and sigma modification parameter. Finally, the paper concludes in Sec. VI.

\section{Wing Rock Dynamics and Adaptive Control}

\section{A. Wing Rock Model}

The model of wing rock, taken from [17], considering a flat, thin wing constrained such that it is free only to roll about its $\mathrm{X}$-axis, is described as

$$
\ddot{\phi}=\left(\rho U_{\infty}^{2} S b / 2 I_{x x} C_{l}\right)+d_{0} u
$$

where $\phi$ is the roll angle and the overdot denotes time derivative. $d_{0}$ is the control effectiveness element and the input signal $u$ acts as part of the angular acceleration. The roll-moment coefficient is written as

$$
C_{l}=a_{1} \phi+a_{2} \dot{\phi}+a_{3} \phi^{3}+a_{4} \phi^{2} \dot{\phi}+a_{5} \phi \dot{\phi}^{2}
$$

The aerodynamic parameters $a_{i}$ are nonlinear functions of the angle of attack which have been presented in [17]

Defining $x=\left(x_{1}, x_{2}\right)^{T}=(\phi, \dot{\phi})^{T}$, the wing rock system can be cast as

$$
\left\{\begin{array}{l}
\dot{x}_{1}=x_{2} \\
\dot{x}_{2}=f(x)+d_{0} u
\end{array}\right.
$$

where $x \in \mathbb{R}^{2}$, the Euclidean space of dimension of two,

$$
f(x)=b^{T} h(x)
$$


and

$$
\begin{gathered}
b=\left[b_{1}, b_{2}, b_{3}, b_{4}, b_{5}\right]^{T}, b_{i}=\left(\rho U_{\infty}^{2} S b / 2 I_{x x}\right) a_{i} \quad i=1, \ldots, 5 \\
h(x)=\left[x_{1}, x_{2}, x_{1}^{3}, x_{1}^{2} x_{2}, x_{1} x_{2}^{2}\right]^{T}
\end{gathered}
$$

\section{B. Model Reference Adaptive Control}

A model reference adaptive controller is applied to suppress the wing rock motion. The reference model carrying the desired response is given by

$$
\dot{x}_{m}=A_{m} x_{m}
$$

where $x_{m}=\left[x_{m 1}, x_{m 2}\right]^{T}, \varsigma>0, \omega_{n}>0$ and

$$
A_{m}=\left[0,1 ;-\omega_{n}^{2},-2 \varsigma \omega_{n}\right]
$$

The reference signal is chosen as $x_{m}(t)=0$ in this study. The desired damping $\varsigma$ and natural frequency $\omega_{n}$ are 0.707 and 0.5 , respectively.

The wing rock control problem is formed under the assumption on the function $f(x)$ : suppose that in Eq. (3) the parameters $b_{i}, i=1, \ldots, 5$ and $d_{0}$ are unknown, but the sign of $d_{0}$ is known.

Defining the tracking error by

$$
\dot{e}=A_{m} e+d_{m}\left[\left(b+b_{m}\right)^{T} h(x)+d_{0} u\right]
$$

where $d_{m}=[0,1]^{T}$ and $b_{m}=\left[\omega_{n}{ }^{2}, 2 \varsigma \omega_{n}, 0,0,0\right]^{T}$. Then the control law is chosen as

$$
u=-\theta^{T} h(x)
$$

where $\theta=\left[\theta_{1}, \theta_{2}, \theta_{3}, \theta_{4}, \theta_{5}\right]^{T}$ is a vector of adaptation parameters to enable the control input to cancel the system uncertainty. Then the adaptation law governing the behavior of parameter vector $\theta$ is defined as

$$
\dot{\theta}=\operatorname{sgn}\left(d_{0}\right) \Gamma\left(e^{T} P d_{m}\right) h(x)-\kappa_{R} \Gamma \theta
$$

where $d_{m}=[0,1]^{T}$; the tracking error $e$ represents the difference between the state $x$ and the reference signal $x_{m}$. The adaptation gain $\Gamma=\operatorname{diag}\left(\Gamma_{i}\right), i=1, \ldots, 5$ with $\Gamma_{i}>0$ and sigma modification gain $\kappa_{R}$ are two important parameters to tune. The adaptation gain determines how quickly $\theta$ evolves, and the sigma modification gain helps to prevent the parameter drift by ensuring $\theta$ bounded, which adds robustness to the system. $P$ is the positive definite solution of the Lyapunov equation

$$
A^{T} P+P A=-Q, Q>0
$$

where $A=A_{m}$. The stability analysis is given in $[27,28]$.

\section{Disturbed Adaptive Control}

Before entry into service, the implementation of controllers must be verified and validated under certain circumstances including modeling error, time delay, uncertainty, and disturbance. In terms of disturbance, a common scenario where the signal measurement process inevitably interferes is considered. The disturbance is then introduced during the measurement of roll angle $\phi$ and angular acceleration $\dot{\phi}$. Here, it is denoted as $d_{d}$ and takes a quadratic form

$$
d_{d}=\mu x_{2}^{2}
$$

where the constant $\mu$ indicates the level of the disturbance.

The disturbed measurement signals are written as

$$
x_{d}=\left[x_{1 d}, x_{2 d}\right]^{T}=\left[x_{1}+d_{d}, x_{2}+d_{d}\right]^{T}
$$

which is used to construct control input signal

$$
u_{d}=-\theta^{T} h_{d}\left(x_{d}\right)
$$

where $u_{d}$ is the actual signal sent to the executing agency, and $h_{d}$ is the relevant five-vector function. In this case, the actual adaptation law applied to the wing rock system becomes

$$
\dot{\theta}=-\operatorname{sgn}\left(d_{0}\right) \Gamma\left(e^{T} P d_{m}\right) \cdot h_{d}\left(x_{d}\right)-\kappa_{R} \Gamma \theta
$$

and the error dynamics

$$
\dot{e}=A_{m} e+d_{m}\left\{d_{0} \tilde{\theta} h_{d}\left(x_{d}\right)+\left(b-b_{m}\right)\left[h(x)-h_{d}\left(x_{d}\right)\right]\right\}
$$


It shows that the control input cannot cancel the part of the uncertainty so that the system cannot guarantee asymptotical stability. It indicates that the disturbance affects the original stability of the system. Thus, the information of the region of attraction for the origin is necessary to evaluate whether the operative range is still included in the stable range, which helps to provide confidence for the implementation of the controller.

The aerodynamic parameters for an angle of attack of $25 \mathrm{deg}$ [27] are used in this paper for the demonstration of the occurrence and suppression of wing rock. The dimensionless time $t^{*}=\left(4 U_{\infty} / b\right) t$ as in [27] is also introduced. Parameters in Eq. (3) are listed below with $t^{*}$ as an independent variable rather than $t$

$$
b_{1}=-0.02012844, \quad b_{2}=0.01051916
$$

$$
\begin{gathered}
b_{3}=0.02596236, \quad b_{4}=-0.1273338, \quad b_{5}=0.5197074 \\
d_{0}=1
\end{gathered}
$$

In Fig. 1, the wing rock phenomenon occurs at a smaller initial condition which shows as a limit cycle on the $\phi-\dot{\phi}$ plane. But the aircraft loses stability at larger conditions. For the convenience of explanation, the disturbance level $\mu$ is specified to take the value of 0.1. Simulation of the response is similar to that of the undisturbed control system for the two smaller initial conditions as shown in Fig. 2, where the wing rock phenomena are successfully suppressed. This corresponds to the equilibrium analysis of the closed-loop system (13) and (14), which indicates that the origin is locally asymptotically stable with eigenvalues $-0.3535+0.3536$ i. However, random trajectory simulation shows that the roll angle and angular acceleration go into infinity under some initial conditions, for example, the largest initial condition in Fig. 2. Therefore, the ROA needs to be evaluated.

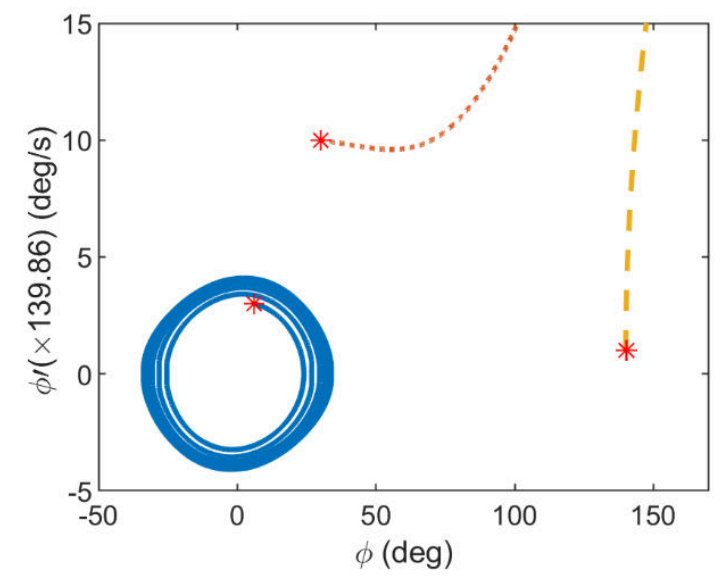

Fig. 1 Phase portrait for the open-loop wing rock system 


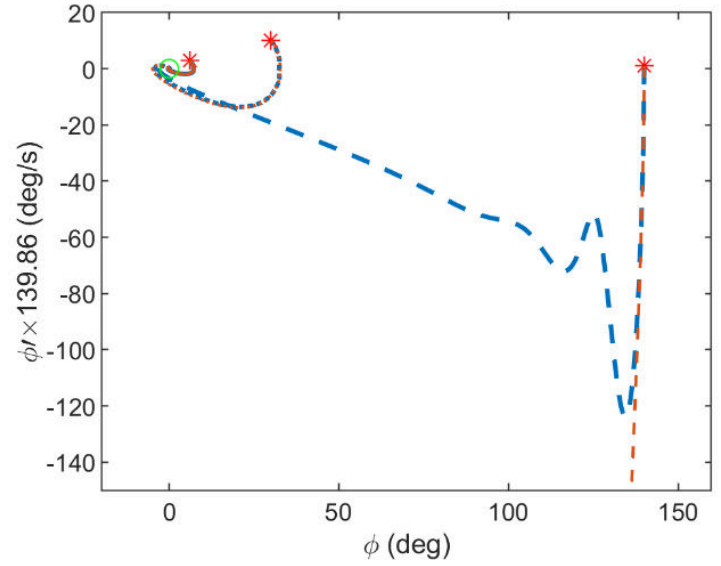

a)

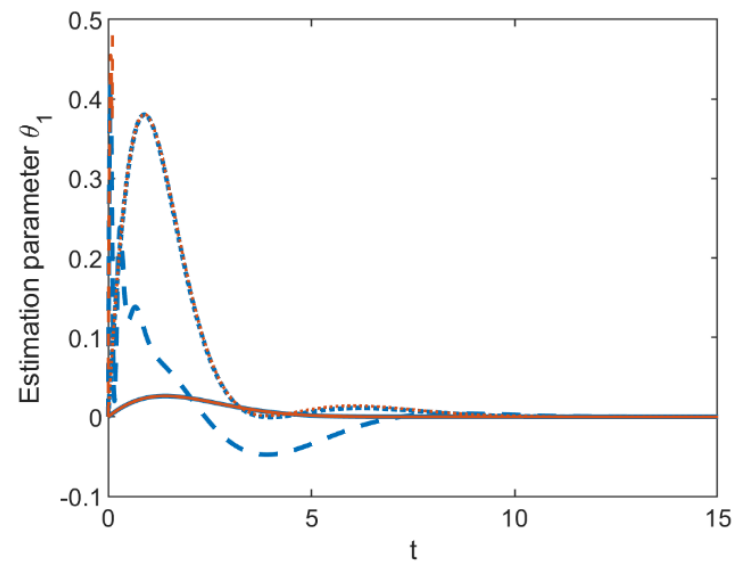

b)

Fig. 2 Phase portrait for adaptive wing rock system with (red) and without disturbance (blue) measurement error

\section{Region of Attraction Estimation}

\section{A. Lyapunov Theory}

Consider an autonomous nonlinear polynomial system of the form

$$
\dot{x}=F(x), \quad x(0)=x_{0}
$$

with $F: \mathbb{R}^{n} \rightarrow \mathbb{R}^{n}$ and $x \in \mathbb{R}^{n}$. Without loss of generality, we assume that the origin is an asymptotically stable equilibrium point such that $f(0)=0$. The ROA, a set of initial conditions whose trajectories will always converge back to the origin, can be defined as

$$
\Omega:=\left\{x_{0} \in \mathbb{R}^{n}: \text { If } x(0)=x_{0} \text { then } \lim _{t \rightarrow \infty} x(t)=0\right\}
$$

Based on the proved lemma which follows the direct Lyapunov theory that specifies a sublevel set of a Lyapunov function as an estimation of the ROA for an asymptotically stable equilibrium point, the compact set $\Omega$ is estimated.

Lemma 1 If there exist a continuously differentiable scalar function $V(x): \mathbb{R}^{n} \rightarrow \mathbb{R}$ and a positive scalar $\gamma \in \mathbb{R}^{+}$, such that

$$
\begin{aligned}
& V(x)>0 \quad \forall x \neq 0 \text { and } V(0)=0 \\
& \Omega_{\gamma}:=\{x: V(x) \leq \gamma\} \text { is bounded } \\
& \Omega_{\gamma} \subseteq\{x:(\partial V / \partial x) F<0\} \cup\{0\}
\end{aligned}
$$

then the origin is asymptotically stable and $\Omega_{\gamma}$ is a subset of the ROA. $V(x)$ is the so-called local Lyapunov function.

\section{B. Sum of Squares (SOS) Techniques}

Making use of the SOS polynomial, the hard problem of a multivariable polynomial being non-negative can be relaxed to being the sum of squares. Then the inequalities as well as set containment conditions in Eqs. (17)-(19) become tractable in the form of an SOS problem. Several toolboxes, such as SOSTOOLs, SOSOPTs, and solvers, such as SeDuMi[9], have been distributed and are freely available for solving such problems. For example, verifying a polynomial $s(x) \in \boldsymbol{R}(x)$ ( $\boldsymbol{R}(x)$ represents the set of polynomials in $x \in \mathbb{R}^{n}$ with real coefficients) being an SOS polynomial is equivalent to checking the existence of a positive semidefinite matrix $Q$, such that

$$
s(x)=Z^{T}(x) Q Z(x) \in \Sigma_{n}
$$

where $Z(x)$ is the properly chosen vector of monomials and $\Sigma_{n}$ denotes the set of SOS polynomial in $x \in \mathbb{R}^{n}$. This is the basic problem in SOS programming.

Besides, the well-known generalized S-procedure[14] is employed to provide sufficient conditions for the set containment condition. 
Lemma 2 (Generalized S-procedure). Given polynomials $g_{0}(x), \ldots, g_{m}(x) \in \boldsymbol{R}[x]$ and polynomials $s_{1}(x), \ldots, s_{m}(x) \in \Sigma_{n}$, if

$$
g_{0}(x)-\sum_{i=1}^{m} s_{i}(x) g_{i}(x) \geq 0
$$

then

$$
\left\{x \mid g_{1}(x), \ldots, g_{m}(x) \geq 0\right\} \subseteq\left\{x \mid g_{0}(x) \geq 0\right\}
$$

Then we can approximate the set (16) by

$$
\begin{gathered}
\max _{V \in \Sigma_{n}, s_{1} \in \Sigma_{n}} \gamma \\
\text { Subject to :-[( } \left.-[V / \partial x) F+l_{1}\right]-(\gamma-V) s_{1} \in \Sigma_{n}
\end{gathered}
$$

where $l_{1}$ is a small positive polynomial (typically $\varepsilon x^{T} x$ with some small real number $\varepsilon$ ) which is used to guarantee the derivative of $V$ to be strictly negative. $s_{1}(x)$ is an SOS multiplier with a proper degree. According to [29], the degree can be chosen by

$$
\begin{aligned}
\max D\left(V \cdot s_{1}\right) & \geq \max D\left((\partial V / \partial x) F+l_{1}\right) \\
\min D\left(s_{1}\right) & \geq \min D\left((\partial V / \partial x) F+l_{1}\right)
\end{aligned}
$$

where $D\left(s_{1}\right)$ represents the degree of the polynomial $s_{1}$. A proper choice of $s_{1}$ is important to capture the feasible result of the problem described by Eq. (23) without introducing unnecessary computation burden. Because a higher degree of $s_{1}$ will introduce more decision variables.

Moreover, the problem (23) is bilinear because the decision variables in $V$ and $s_{1}$ are coupled, and also the variable to be optimized $\gamma$ is not affine. However, due to the fact that the linear solver has more advantages than the nonlinear ones such as PENMBI in YALMIP[30], (23) has to be modified to accommodate to linear solver used in this paper. It is decoupled by choosing a feasible Lyapunov function $V$ in advance and keeping it fixed; the optimized $\gamma$ is searched by bisection procedure.

A Lyapunov function can be constructed using its physical meaning for a specific problem. While a systematic way is employed here to generate an initial choice

$$
V=x^{T} P x
$$

where $P$ is the solution of Lyapunov equation (9) with $A=\left.(\partial F / \partial x)\right|_{x=0}$ that is assumed to be Hurwitz. In [15], it is explained why $Q=I$ is chosen but it is still a conservative choice since only the largest ball is provided. Other positive definite $Q$ can also be customized to adapt to a specific shaped ROA when prior knowledge is available.

The optimized $\gamma$ provides a lower bound for the ROA estimation. Then exhaustive Monte Carlo search is carried out using $\theta_{i}=0(i=1, \ldots, 5)$ to give an insight into the ROA on the $x_{1}-x_{2}$ plane.

\section{Results}

The parameter update law in the MRAC is tuned by adjusting the adaptation rate $\Gamma$ and sigma modification $\kappa_{R}$. As mentioned before, the sigma modification is employed to ensure $\theta$ remains bounded. Generally, the system displays increased performance in tighter tracking and faster rise time as the adaptation rate increases. However, it has been shown that increasing $\Gamma$ will otherwise bring a decrease in system robustness[1]. Therefore, it is necessary to gain more insights into the impact of these parameters on system performance. Especially with the introduced disturbance, how does the choice of these parameters influence the ROA of the control system? Should these parameters be redesigned to ensure the aircraft system operates normally under such a scenario? In the following parts, the problem is explored through ROA estimation of the disturbed adaptive system.

To initialize the bisection procedure for estimation, a first Lyapunov function is computed as

$$
V_{0}=x^{T} P x+\theta^{T} \Gamma^{-1} \theta
$$

The lower bound for $\gamma$ is set zero and an upper bound can be determined by trial and error. A level set is obtained when tolerance $\left|\left(\gamma_{i}-\gamma_{i-1}\right) / \gamma_{i-1}\right| \leq 1 \mathrm{e}-3$ is reached.

\section{A. Adaptation Rate}


The effect of varying adaptation rates is explored by analyzing the ROA with respect to the origin of the disturbed adaptive system. In this case, the sigma modification value is held constant as 1 to isolate the effect of changing adaptation rate. The ROA variation trends are shown in Fig. 3.
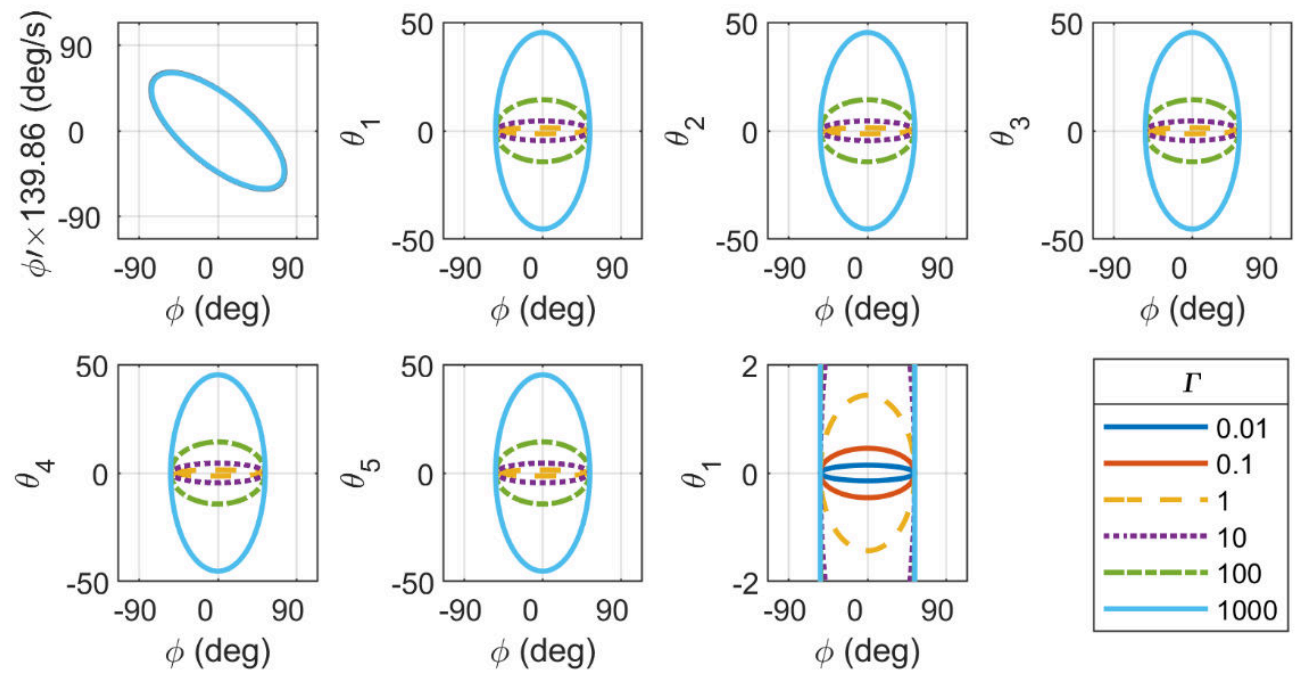

Fig. 3 ROA variation with adaptation rate

The results obtained by the SOS method give a lower bound of the actual ROA of the system. As shown in Fig. 3, the valid region on the $\phi-\dot{\phi}$ plane hardly changes with the variation of adaptation rate $\Gamma$, but the bounds for $\theta_{i}$ grow as the adaptation rate increases. This result is intuitive as an increasing $\Gamma$ leads to a fast adaptation of the unknown parameters $\theta_{i}$ and brings them to a steady value quickly thus increasing the robustness of the system, which shows a larger ROA. It suggests that a larger adaptation rate can stabilize the system on a wider range of initial conditions.

\section{B. Sigma Modification}

The effect of varying sigma modification values on the MRAC closed-loop system under certain measurement errors is explored by analyzing the region of attraction around the origin.
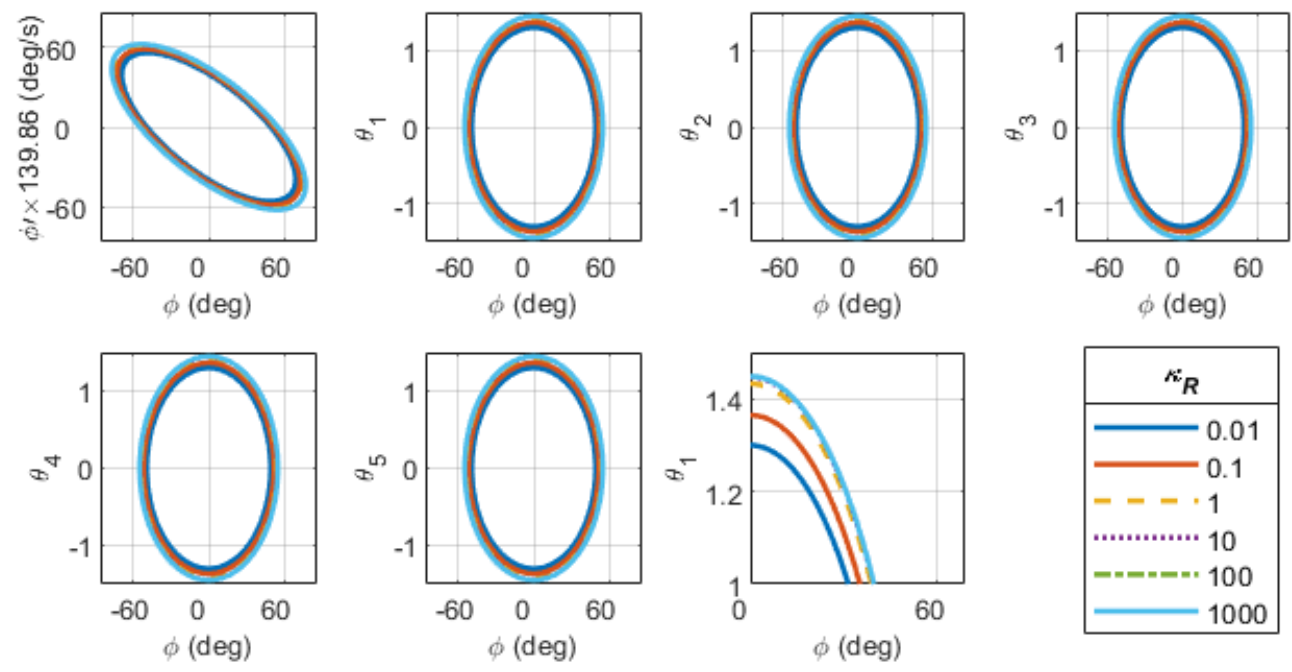

Fig. 4 ROA variation with sigma modification value

With the adaptation rate fixed at $\Gamma=I$, the ROA estimation with respect to various sigma modification values is shown in Fig. 4. The ROA expands slightly as $\kappa_{R}$ increases. The sigma modification is used to prevent parameter 
drift by bounding the evaluation of estimation parameters. Trajectory simulation shows that the larger the sigma modification value, the faster the convergence and the smaller the overshoot. This can bound the trajectories in a reasonable small range of variation thus is beneficial for the whole system, and correspondingly, can stabilize a larger range of initial conditions. The illustrative results by the SOS method in Fig. 4 reveal this characteristic.

\section{Conclusion}

The region of attraction(ROA) estimation using sum of squares(SOS) optimization technique is used for nonlinear analysis for the adaptive control system. The ROA is computed with respect to varying control design parameters, the adaptation rate and sigma modification values, and then their effects on ROA estimation are evaluated. Though conservative, the results are illustrative in revealing the nonlinear characteristics of the adaptive control system and in giving insights into the controller design. The SOS method provides guarantees on the certification process of control systems.

\section{Acknowledgments}

This work was supported by Cranfield University; the China Scholarship Council under Grant No. 201906840024; and the Fundamental Research Funds for the Central Universities, China, No. 30919011401.

\section{References}

[1] Dydek, Z. T., Jain, H., Jinho, J., Annaswamy, A. A. M., Lavretsky, E., Jang, J., Annaswamy, A. A. M., and Lavretsky, E. "Theoretically Verifiable Stability Margins for an Adaptive Controller," AIAA Guidance, Navigation, and Control Conference 2006, Vol. 5, American Institute of Aeronautics and Astronautics, Keystone, Colorado, 2006, pp. $2821-2834$. https://doi.org/10.2514/6.2006-6416.

[2] Chandramohan, R., and Calise, A. J. "Output Feedback Adaptive Control of Plants with Unmodeled Dynamics and Input Uncertainties." Journal of Guidance, Control, and Dynamics, Vol. 42, No. 10, 2019, pp. 2143-2156. https://doi.org/10.2514/1.G003548.

[3] Huang, C., and Yu, C. "Global Adaptive Controller for Linear Systems with Unknown Input Delay.” IEEE Transactions on Automatic Control, Vol. 62, No. 12, 2017, pp. 6589-6594. https://doi.org/10.1109/TAC.2017.2714858.

[4] Dobrokhodov, V., Kaminer, I., Kitsios, I., Xargay, E., Hovakimyan, N., Cao, C., Gregory, I. M., and Valavani, L. "Experimental Validation of L1 Adaptive Control: The Rohrs Counterexample in Flight." Journal of Guidance, Control, and Dynamics, Vol. 34, No. 5, 2011, pp. 1311-1328. https://doi.org/10.2514/1.50683.

[5] Jacklin, S., Schumann, J., Gupta, P., Lowry, M., Bosworth, J., Zavala, E., Hayhurst, K., Belcastro, C., and Belcastro, C. "Verification, Validation, and Certification Challenges for Adaptive Flight-Critical Control System Software," AIAA Guidance, Navigation, and Control Conference and Exhibit, Vol. 4, American Institute of Aeronautics and Astronautics, Reston, Virigina, 2004, pp. 2648-2657. https://doi.org/10.2514/6.2004-5258.

[6] Dorobantu, A., Seiler, P., and Balas, G. J. "Time-Delay Margin Analysis for an Adaptive Controller." Journal of Guidance, Control, and Dynamics, Vol. 35, No. 5, 2012, pp. 1418-1425. https://doi.org/10.2514/1.56719.

[7] Amato, F., Calabrese, F., Cosentino, C., and Merola, A. "Stability Analysis of Nonlinear Quadratic Systems via Polyhedral Lyapunov Functions.” Automatica, Vol. 47, No. 3, 2011, pp. 614-617. https://doi.org/10.1016/j.automatica.2010.12.005.

[8] Topcu, U., Packard, A., and Seiler, P. "Local Stability Analysis Using Simulations and Sum-of-Squares Programming." Automatica, Vol. 44, No. 10, 2008, pp. 2669-2675. https://doi.org/10.1016/j.automatica.2008.03.010.

[9] Chakraborty, A., Seiler, P., and Balas, G. J. G. J. G. J. "Nonlinear Region of Attraction Analysis for Flight Control Verification and Validation." Control Engineering Practice, Vol. 19, No. 4, 2011, pp. 335-345. https://doi.org/10.1016/j.conengprac.2010.12.001.

[10] Cunis, T., Condomines, J.-P. P., and Burlion, L. "Local Stability Analysis for Large Polynomial Spline Systems." Automatica, Vol. 113, 2020, pp. 1-5. https://doi.org/10.1016/j.automatica.2019.108773.

[11] Azam, M., and Singh, S. N. "Invertibility and Trajectory Control for Nonlinear Maneuvers of Aircraft." Journal of Guidance, Control, and Dynamics, Vol. 17, No. 1, 1994, pp. 192-200. https://doi.org/10.2514/3.21178.

[12] Sidorov, E., and Zacksenhouse, M. "Lyapunov Based Estimation of the Basin of Attraction of Poincare Maps with Applications to Limit Cycle Walking.” Nonlinear Analysis: Hybrid Systems, Vol. 33, 2019, pp. 179-194. https://doi.org/10.1016/j.nahs.2019.03.002.

[13] Genesio, R., Tartaglia, M., and Vicino, A. "On the Estimation of Asymptotic Stability Regions: State of the Art and New Proposals." IEEE Transactions on Automatic Control, Vol. 30, No. 8, 1985, pp. 747-755. https://doi.org/10.1109/TAC.1985.1104057.

[14] Topcu, U., and Packard, A. "Local Stability Analysis for Uncertain Nonlinear Systems." IEEE Transactions on Automatic Control, Vol. 54, No. 5, 2009, pp. 1042-1047. https://doi.org/10.1109/TAC.2009.2017157.

[15] Khodadadi, L., Samadi, B., and Khaloozadeh, H. "Estimation of Region of Attraction for Polynomial Nonlinear Systems: A Numerical Method.” ISA Transactions, Vol. 53, No. 1, 2014, pp. 25-32. https://doi.org/10.1016/j.isatra.2013.08.005. 
[16] Papachristodoulou, A., and Prajna, S. “A Tutorial on Sum of Squares Techniques for Systems Analysis," Proceedings of the 2005, American Control Conference, 2005., Vol. 4, IEEE, Portland, OR, USA, 2005, pp. 2686-2700. https://doi.org/10.1109/ACC.2005.1470374.

[17] Nayfeh, A. H., Elzebda, J. M., and Mook, D. T. "Analytical Study of the Subsonic Wing-Rock Phenomenon for Slender Delta Wings.” Journal of Aircraft, Vol. 26, No. 9, 1989, pp. 805-809. https://doi.org/10.2514/3.45844.

[18] Elzebda, J. M., Nayfeh, A. H., and Mook, D. T. "Development of an Analytical Model of Wing Rock for Slender Delta Wings." Journal of Aircraft, Vol. 26, No. 8, 1989, pp. 737-743. https://doi.org/10.2514/3.45833.

[19] Cao, C., Hovakimyan, N., and Lavretsky, E. “Application of L1 Adaptive Controller to Wing Rock," AIAA Guidance, Navigation, and Control Conference and Exhibit, Vol. 5, American Institute of Aeronautics and Astronautics, Keystone, Colorado, 2006, pp. 2941-2952. https://doi.org/10.2514/6.2006-6426.

[20] Parrilo, P. A. "Structured Semidefinite Programs and Semialgebraic Geometry Methods in Robustness and Optimization." California Institute of Technology, Pasadena, CA, 2000.

[21] Tan, W., and Packard, A. "Stability Region Analysis Using Polynomial and Composite Polynomial Lyapunov Functions and Sum-of-Squares Programming.” IEEE Transactions on Automatic Control, Vol. 53, No. 2, 2008, pp. 565-571. https://doi.org/10.1109/TAC.2007.914221.

[22] Chen, Y.-J., Tanaka, M., Tanaka, K., and Wang, H. O. "Stability Analysis and Region-of-Attraction Estimation Using Piecewise Polynomial Lyapunov Functions: Polynomial Fuzzy Model Approach.” IEEE Transactions on Fuzzy Systems, Vol. 23, No. 4, 2015, pp. 1314-1322. https://doi.org/10.1109/TFUZZ.2014.2347993.

[23] Topcu, U., Packard, A. K., Seiler, P., and Balas, G. J. "Robust Region-of-Attraction Estimation." IEEE Transactions on Automatic Control, Vol. 55, No. 1, 2010, pp. 137-142. https://doi.org/10.1109/TAC.2009.2033751.

[24] Chakraborty, A., Seiler, P., and Balas, G. J. "Susceptibility of F/A-18 Flight Controllers to the Falling-Leaf Mode: Nonlinear Analysis." Journal of Guidance, Control, and Dynamics, Vol. 34, No. 1, 2011, pp. 73-85. https://doi.org/10.2514/1.50675.

[25] Sidoryuk, M. E., and Khrabrov, A. N. "Estimation of Regions of Attraction of Aircraft Spin Modes." Journal of Aircraft, Vol. 56, No. 1, 2019, pp. 205-216. https://doi.org/10.2514/1.C034936.

[26] Li, D., Ignatyev, D., Tsourdos, A., and Wang, Z. "Region of Attraction Analysis for Adaptive Control of Wing Rock System.” IFAC-PapersOnLine, Vol. 54, No. 14, 2021, pp. 518-523. https://doi.org/10.1016/j.ifacol.2021.10.407.

[27] Singh, S. N., Yirn, W., and Wells, W. R. "Direct Adaptive and Neural Control of Wing-Rock Motion of Slender Delta Wings.” Journal of Guidance, Control, and Dynamics, Vol. 18, No. 1, 1995, pp. 25-30. https://doi.org/10.2514/3.56652.

[28] Calise, A. J., Shin, Y., and Johnson, M. D. "A Comparison Study of Classical and Neural Network Based Adaptive Control of Wing Rock.” AIAA Guidance, Navigation, and Control Conference, Vol. 4, No. August, 2004, pp. $2728-2744$. https://doi.org/10.2514/6.2004-5320.

[29] Weehong Tan, and Packard, A. "Stability Region Analysis Using Sum of Squares Programming," 2006 American Control Conference, Vol. 2006, IEEE, 2006, p. 6 pp. https://doi.org/10.1109/ACC.2006.1656562.

[30] Tibken, B., Youping Fan, and Fan, Y. "Computing the Domain of Attraction for Polynomial Systems via BMI Optimization Method." Proceedings of the American Control Conference, Vol. 2006, 2006, pp. 117-122. https://doi.org/10.1109/acc.2006.1655340. 
2021-12-29

Nonlinear analysis for wing rock system with adaptive control

Li, Dongyang

AIAA

Li D, Ignatyev D, Tsourdos A, Wang Z. (2021) Nonlinear analysis for wing rock system with adaptive control. In: AIAA SciTech 2022 Forum, 3-7 January 2022, San Diego, USA and Virtual Event https://doi.org/10.2514/6.2022-0691

Downloaded from Cranfield Library Services E-Repository 